\title{
PROOF OF A CONJECTURED ASYMPTOTIC EXPANSION FOR THE APPROXIMATION OF SURFACE INTEGRALS
}

\author{
P. VERLINDEN AND R. COOLS
}

\begin{abstract}
Georg introduced a new kind of trapezoidal rule and midpoint rule to approximate a surface integral over a curved triangular surface and conjectured the existence of an asymptotic expansion for this approximation as the subdivision of the surface gets finer. The purpose of this paper is to prove the conjecture.
\end{abstract}

\section{INTRODUCTION}

In [1] Georg introduced a new kind of trapezoidal rule and midpoint rule to approximate a surface integral over a curved triangular surface and made a conjecture about the asymptotic behavior of the approximation as the subdivision of the surface gets finer. Recently, Georg and Tausch [2] found a partial proof of this conjecture. Strong numerical evidence supports the validity of the conjecture. The purpose of this paper is to prove the conjecture. Simultaneously and independently, Lyness [3] obtained similar results.

Let $\sigma$ denote the triangle

$$
\sigma:=\left\{(u, v) \in \mathbb{R}^{2}: 0 \leq u, 0 \leq v, u+v \leq 1\right\}
$$

and

$$
V:=\{(0,0),(1,0),(0,1)\}
$$

its vertices. Let $a_{i, j}^{n}$ and $\bar{a}_{i, j}^{n}$ denote the affine maps

$$
a_{i, j}^{n}(u, v):=\left(\frac{u+i}{n}, \frac{v+j}{n}\right), \quad \bar{a}_{i, j}^{n}(u, v):=\left(\frac{1-u+i}{n}, \frac{1-v+j}{n}\right)
$$

and

$$
\mathscr{A}^{n}:=\left\{a_{i, j}^{n}: 0 \leq i+j \leq n-1\right\} \cup\left\{\bar{a}_{i, j}^{n}: 0 \leq i+j \leq n-2\right\} .
$$

Then

$$
\sigma=\bigcup_{\alpha \in \mathscr{A}^{n}} \alpha(\sigma)
$$

represents the regular subdivision of the triangle $\sigma$ into $n^{2}$ subtriangles. Let

$$
\phi: \sigma \rightarrow \mathbb{R}^{3}:(u, v) \mapsto(x, y, z)=\phi(u, v)
$$

Received by the editor June 2, 1993 and, in revised form, December 7, 1993.

1991 Mathematics Subject Classification. Primary 65N38, 65D30.

Key words and phrases. Numerical integration, surface integral, Euler-Maclaurin expansion, boundary element method. 
be the parametrization of a surface $S=\phi(\sigma)$ in $\mathbb{R}^{3}$ and let $g(u, v)$ be a function on $\sigma$. Then we define the operator $\mathscr{S}_{\phi}^{n}$ as

$$
\left(\mathscr{S}_{\phi}^{n} g\right)(u, v):=\sum_{\alpha \in \mathscr{A}^{n}}(g \circ \alpha)(u, v) \operatorname{area}[(\phi \circ \alpha)(V)]
$$

where

$$
\begin{aligned}
& \operatorname{area}[(\phi \circ \alpha)(V)] \\
& \quad:=\frac{1}{2}\|((\phi \circ \alpha)(1,0)-(\phi \circ \alpha)(0,0)) \times((\phi \circ \alpha)(0,1)-(\phi \circ \alpha)(0,0))\|
\end{aligned}
$$

is the area of the triangle with vertices $(\phi \circ \alpha)(V)$ and $\|\cdot\|$ represents the usual Euclidean norm in $\mathbb{R}^{3}$. We use the following notation for partial derivatives:

$$
f^{\left(k_{1}, k_{2}, \ldots, k_{n}\right)}\left(x_{1}, x_{2}, \ldots, x_{n}\right)=\frac{\partial^{k_{1}+k_{2}+\cdots+k_{n}}}{\partial x_{1}^{k_{1}} \partial x_{2}^{k_{2}} \cdots \partial x_{n}^{k_{n}}} f\left(x_{1}, x_{2}, \ldots, x_{n}\right) .
$$

If $g$ is continuous and $\phi$ is continuously differentiable, we have

$$
\lim _{n \rightarrow \infty}\left(\mathscr{S}_{\phi}^{n} g\right)(u, v)=\iint_{\sigma} g(u, v)\left\|\phi^{(1,0)}(u, v) \times \phi^{(0,1)}(u, v)\right\| d u d v .
$$

Hence, if $f$ is a continuous function on $S$,

$$
\left(\mathscr{S}_{\phi}^{n}(f \circ \phi)\right)(u, v)
$$

converges to the surface integral of $f$ over $S$

$$
\int_{S} f d S=\iint_{\sigma} f(\phi(u, v))\left\|\phi^{(1,0)}(u, v) \times \phi^{(0,1)}(u, v)\right\| d u d v .
$$

To approximate this surface integral, Georg and Tausch considered the modified trapezoidal rule

$$
\frac{1}{3}\left(\left(\mathscr{S}_{\phi}^{n}(f \circ \phi)\right)(0,0)+\left(\mathscr{S}_{\phi}^{n}(f \circ \phi)\right)(1,0)+\left(\mathscr{S}_{\phi}^{n}(f \circ \phi)\right)(0,1)\right)
$$

and the modified midpoint rule

$$
\left(\mathscr{S}_{\phi}^{n}(f \circ \phi)\right)\left(\frac{1}{3}, \frac{1}{3}\right) \text {. }
$$

If we transformed the surface integral into an integral over the triangle $\sigma$ and approximated the latter integral numerically, we would have to evaluate the partial derivatives of the map $\phi$, which is only defined implicitly in applications of boundary element methods [1]. The advantage of the rules (3) and (4) is that they avoid the evaluation of these partial derivatives. Georg conjectured that these rules admit an asymptotic expansion in even powers of $\frac{1}{n}$ under certain regularity conditions on $f$ and $\phi$. We prove this conjecture in Theorem 1 .

\section{PROOF OF THE ASYMPTOTIC EXPANSION}

Definition 1. Suppose $\Omega$ is an open subset of $\mathbb{R}^{n}$ and $\Omega$ its closure. Then we say that a function $f$ is smooth on $\Omega$ if the partial derivatives of all orders of $f$ exist and are continuous on $\Omega$ and we say that $f$ is smooth on $\bar{\Omega}$ if $f$ is 
smooth on $\Omega$ and if the partial derivatives of all orders of $f$ have a continuous extension on $\Omega$.

Lemma 1. Suppose $f(x, y)$ is a smooth function on the square $[0,1]^{2}$. Then we have the asymptotic expansion

$$
\begin{aligned}
& \frac{1}{n^{2}} \sum_{i=0}^{n-1} \sum_{j=0}^{n-1} f\left(\frac{\frac{1}{2}+i}{n}, \frac{\frac{1}{2}+j}{n}\right) \\
& \quad \sim \sum_{k=0}^{\infty} \sum_{l=0}^{\infty} \frac{1}{n^{k+l}} \int_{0}^{1} \int_{0}^{1} f^{(k, l)}(x, y) d x d y \frac{B_{k}\left(\frac{1}{2}\right) B_{l}\left(\frac{1}{2}\right)}{k ! l !} \text { as } n \rightarrow \infty,
\end{aligned}
$$

where $B_{m}(t)$ represents the Bernoulli polynomial of degree $m$ in $t$.

This is a well-known generalization of the classical Euler-Maclaurin summation formula (cf. [4]). Observe that $B_{m}\left(\frac{1}{2}\right)=0$ if $m$ is odd. Consequently, we have an asymptotic expansion in even powers of $\frac{1}{n}$. The following lemma combines this asymptotic expansion with a Taylor expansion.

Lemma 2. Suppose the function $f(x, y, h)$ is smooth on

$$
\bar{\Omega}=\left\{(x, y, h) \in \mathbb{R}^{3}: 0 \leq h \leq \epsilon, \frac{h}{2} \leq x \leq 1-\frac{h}{2}, \frac{h}{2} \leq y \leq 1-\frac{h}{2}\right\},
$$

where $\epsilon>0$. Then we have the asymptotic expansion

$$
\begin{aligned}
& \frac{1}{n^{2}} \sum_{i=0}^{n-1} \sum_{j=0}^{n-1} f\left(\frac{\frac{1}{2}+i}{n}, \frac{\frac{1}{2}+j}{n}, \frac{1}{n}\right) \\
& \quad \sim \sum_{k=0}^{\infty} \sum_{l=0}^{\infty} \sum_{m=0}^{\infty} \frac{1}{n^{k+l+m}} \int_{0}^{1} \int_{0}^{1} f^{(k, l, m)}(x, y, 0) d x d y \frac{E_{k}\left(\frac{1}{2}\right) B_{l}\left(\frac{1}{2}\right)}{k ! l ! m !} \text { as } n \rightarrow \infty .
\end{aligned}
$$

Remark. If $f(x, y, h)$ is smooth on

$$
\{(x, y, h):(x, y, h) \in \bar{\Omega} \text { or }(x, y,-h) \in \bar{\Omega}\},
$$

and if $f$ is even with respect to $h$,

$$
f(x, y,-h)=f(x, y, h),
$$

then we have an asymptotic expansion in even powers of $\frac{1}{n}$.

Proof of Lemma 2. Let $M$ be an arbitrary positive integer. Expanding $f(x, y, h)$ in its Taylor series about $h=0$, we have

$$
f(x, y, h)=\sum_{m=0}^{M} f^{(0,0, m)}(x, y, 0) \frac{h^{m}}{m !}+f_{M}(x, y, h) .
$$

The remainder $f_{M}(x, y, h)$ is uniformly of order $\mathscr{O}\left(h^{M+1}\right)$ :

$$
\forall(x, y, h) \in \bar{\Omega}:\left|f_{M}(x, y, h)\right| \leq K h^{M+1},
$$


where

$$
K=\max _{(x, y, h) \in \Omega}\left|\frac{1}{(M+1) !} f^{(0,0, M+1)}(x, y, h)\right| .
$$

Using (5), we have

$$
\begin{aligned}
\frac{1}{n^{2}} \sum_{i=0}^{n-1} & \sum_{j=0}^{n-1} f\left(\frac{\frac{1}{2}+i}{n}, \frac{\frac{1}{2}+j}{n}, \frac{1}{n}\right) \\
= & \sum_{m=0}^{M} \frac{1}{n^{2}} \sum_{i=0}^{n-1} \sum_{j=0}^{n-1} f^{(0,0, m)}\left(\frac{\frac{1}{2}+i}{n}, \frac{\frac{1}{2}+j}{n}, 0\right) \frac{1}{m ! n^{m}} \\
& +\frac{1}{n^{2}} \sum_{i=0}^{n-1} \sum_{j=0}^{n-1} f_{M}\left(\frac{\frac{1}{2}+i}{n}, \frac{\frac{1}{2}+j}{n}, \frac{1}{n}\right) .
\end{aligned}
$$

By Lemma 1, we have

$$
\begin{aligned}
\frac{1}{n^{2+m}} & \sum_{i=0}^{n-1} \sum_{j=0}^{n-1} \frac{1}{m !} f^{(0,0, m)}\left(\frac{\frac{1}{2}+i}{n}, \frac{\frac{1}{2}+j}{n}, 0\right) \\
= & \sum_{k+l+m \leq M} \frac{1}{n^{k+l+m}} \int_{0}^{1} \int_{0}^{1} f^{(k, l, m)}(x, y, 0) d x d y \frac{B_{k}\left(\frac{1}{2}\right) B_{l}\left(\frac{1}{2}\right)}{k ! l ! m !} \\
& +\mathscr{O}\left(\frac{1}{n^{M+1}}\right)
\end{aligned}
$$

and by (6) we have

$$
\left|\frac{1}{n^{2}} \sum_{i=0}^{n-1} \sum_{j=0}^{n-1} f_{M}\left(\frac{\frac{1}{2}+i}{n}, \frac{\frac{1}{2}+j}{n}, \frac{1}{n}\right)\right| \leq \frac{K}{n^{M+1}} .
$$

Substituting (8) and (9) in (7), we have

$$
\begin{aligned}
\frac{1}{n^{2}} \sum_{i=0}^{n-1} & \sum_{j=0}^{n-1} f\left(\frac{\frac{1}{2}+i}{n}, \frac{\frac{1}{2}+j}{n}, \frac{1}{n}\right) \\
= & \sum_{k+l+m \leq M} \frac{1}{n^{k+l+m}} \int_{0}^{1} \int_{0}^{1} f^{(k, l, m)}(x, y, 0) d x d y \frac{B_{k}\left(\frac{1}{2}\right) B_{l}\left(\frac{1}{2}\right)}{k ! l ! m !} \\
& +\mathscr{O}\left(\frac{1}{n^{M+1}}\right) .
\end{aligned}
$$

As $M$ can be chosen arbitrarily large, the lemma is proved.

Lemma 3. Suppose $\phi: \sigma \rightarrow \mathbb{R}^{3}$ is a smooth map such that

$$
\forall(u, v) \in \sigma: \phi^{(1,0)}(u, v) \times \phi^{(0,1)}(u, v) \neq(0,0,0) .
$$


Then there exists an $\epsilon>0$ such that the function

$$
A(u, v, h):=h^{-2} \text { area }\left[\phi\left(\left\{V_{0}(u, v, h), V_{1}(u, v, h), V_{2}(u, v, h)\right\}\right)\right],
$$

where

$$
\begin{aligned}
& V_{0}(u, v, h)=\left(u-\frac{h}{2}, v-\frac{h}{2}\right), \\
& V_{1}(u, v, h)=\left(u+\frac{h}{2}, v-\frac{h}{2}\right), \\
& V_{2}(u, v, h)=\left(u-\frac{h}{2}, v+\frac{h}{2}\right),
\end{aligned}
$$

is smooth on

$$
\bar{\Omega}=\left\{(u, v, h) \in \mathbb{R}^{3}:|h| \leq \epsilon, \frac{|h|}{2} \leq u, \frac{|h|}{2} \leq v, u+v \leq 1-|h|\right\} .
$$

The inequalities in the definition of $\bar{\Omega}$ ensure that $\phi$ is defined at $V_{0}(u, v, h)$, $V_{1}(u, v, h)$ and $V_{2}(u, v, h)$.

Proof of Lemma 3. Let

$$
\begin{aligned}
N(u, v, h)= & \left(\phi\left(V_{1}(u, v, h)\right)-\phi\left(V_{0}(u, v, h)\right)\right) \\
& \times\left(\phi\left(V_{2}(u, v, h)\right)-\phi\left(V_{0}(u, v, h)\right)\right) .
\end{aligned}
$$

Then $N(u, v, h)$ depends smoothly on $(u, v, h)$. Expanding $N(u, v, h)$ in a Taylor series about $h=0$, we have uniformly

$$
N(u, v, h) \sim \sum_{j=0}^{\infty} c_{j}(u, v) h^{j+2} \text { as } h \rightarrow 0,
$$

where

$$
c_{0}(u, v)=\phi^{(1,0)}(u, v) \times \phi^{(0,1)}(u, v) .
$$

The Taylor expansion of $N^{(k, l, m)}(u, v, h)$ is obtained by termwise differentiation of this expansion

$$
N^{(k, l, m)}(u, v, h) \sim \sum_{j=0}^{\infty} c_{j}^{(k, l)}(u, v) \frac{d^{m} h^{j+2}}{d h^{m}} \text { as } h \rightarrow 0 .
$$

Let

$$
M(u, v, h)=h^{-2} N(u, v, h) .
$$

Then, applying the Leibniz rule for differentiation of a product, and using the 
Taylor expansions of the partial derivatives of $N(u, v, h)$, we have

$$
\begin{aligned}
M^{(k, l, m)}(u, v, h)=\sum_{i=0}^{m}\left(\begin{array}{c}
m \\
i
\end{array}\right)\left(\frac{d^{i} h^{-2}}{d h^{i}}\right) N^{(k, l, m-i)}(u, v, h) \\
\quad \sim \sum_{i=0}^{m}\left(\begin{array}{c}
m \\
i
\end{array}\right)\left(\frac{d^{i} h^{-2}}{d h^{i}}\right) \sum_{j=0}^{\infty} c_{j}^{(k, l)}(u, v)\left(\frac{d^{m-i} h^{2+j}}{d h^{m-i}}\right) \text { as } h \rightarrow 0 \\
\quad \sim \sum_{j=0}^{\infty} c_{j}^{(k, l)}(u, v) \sum_{i=0}^{m}\left(\begin{array}{c}
m \\
i
\end{array}\right)\left(\frac{d^{i} h^{-2}}{d h^{i}}\right)\left(\frac{d^{m-i} h^{2+j}}{d h^{m-i}}\right) \text { as } h \rightarrow 0 \\
\sim \sum_{j=0}^{\infty} c_{j}^{(k, l)}(u, v) \frac{d^{m} h^{j}}{d h^{m}} \text { as } h \rightarrow 0 \\
\quad \sim \sum_{j=m}^{\infty} c_{j}^{(k, l)}(u, v) j(j-1) \cdots(j-m+1) h^{j-m} \text { as } h \rightarrow 0,
\end{aligned}
$$

uniformly. Hence, $M(u, v, h)$ admits a smooth extension as $h \rightarrow 0$. As

$$
M(u, v, 0)=\phi^{(1,0)}(u, v) \times \phi^{(0,1)}(u, v),
$$

we have by (10) that

$$
\forall(u, v, h) \in \bar{\Omega}: M(u, v, h) \neq(0,0,0),
$$

provided $\epsilon$ is chosen sufficiently small. As a result,

$$
A(u, v, h)=\frac{1}{2}\|M(u, v, h)\|
$$

is smooth on $\Omega$.

We now state the main result.

Theorem 1. Suppose $\phi$ is as in Lemma 3 and $g(u, v)$ is a smooth function on $\sigma$. Then for $(u, v) \in \sigma$ the following asymptotic expansion holds:

$$
\left(\mathscr{S}_{\phi}^{n} g\right)(u, v) \sim \sum_{k=0}^{\infty} \frac{c_{k}(u, v)}{n^{2 k}} \text { as } n \rightarrow \infty
$$

where $c_{k}(u, v)$ is a polynomial of total degree $\leq 2 k$ in $u$ and $v$.

Proof. We first prove the theorem under the additional assumption

$$
\exists \epsilon_{1}>0: g(u, v)=0 \text { if } u+v \geq 1-\epsilon_{1} .
$$

According to Lemma 3 , there exists an $\epsilon_{2}>0$ such that $A(s, t, h)$ is smooth on

$$
\bar{\Omega}_{2}=\left\{(s, t, h) \in \mathbb{R}^{3}:|h| \leq \epsilon_{2}, \frac{|h|}{2} \leq s, \frac{|h|}{2} \leq t, s+t \leq 1-|h|\right\} .
$$


Choose $0<\epsilon<\frac{1}{2} \min \left\{\epsilon_{1}, \epsilon_{2}\right\}$ and let

$$
\bar{\Omega}=\left\{(s, t, h) \in \mathbb{R}^{3}:|h| \leq \epsilon, \frac{|h|}{2} \leq s \leq 1-\frac{|h|}{2}, \frac{|h|}{2} \leq t \leq 1-\frac{|h|}{2}\right\} .
$$

For each $(s, t, h) \in \bar{\Omega}$, at least one of the following conditions is satisfied:

(i) $s+t+|h|<1$.

If this condition is satisfied, we put

$$
\Gamma(s, t, h):=g\left(s+h\left(u-\frac{1}{2}\right), t+h\left(v-\frac{1}{2}\right)\right) A(s, t, h) .
$$

(ii) $s+t-|h|>1-\epsilon_{1}$.

If this condition is satisfied, we put $\Gamma(s, t, h):=0$.

The conditions are not mutually exclusive, but by (12) both expressions for $\Gamma(s, t, h)$ coincide if both conditions are satisfied. The function $\Gamma(s, t, h)$ is consequently well defined and smooth on $\Omega$. If $n \geq \epsilon^{-1}$, we have

$$
\begin{aligned}
& \left(g \circ a_{i, j}^{n}\right)(u, v) \operatorname{area}\left[\left(\phi \circ a_{i, j}^{n}\right)(V)\right]=\frac{1}{n^{2}} \Gamma\left(\frac{i+\frac{1}{2}}{n}, \frac{j+\frac{1}{2}}{n}, \frac{1}{n}\right), \\
& \left(g \circ \bar{a}_{i, j}^{n}\right)(u, v) \operatorname{area}\left[\left(\phi \circ \bar{a}_{i, j}^{n}\right)(V)\right]=\frac{1}{n^{2}} \Gamma\left(\frac{i+\frac{1}{2}}{n}, \frac{j+\frac{1}{2}}{n},-\frac{1}{n}\right) .
\end{aligned}
$$

Inserting this in (1), we have

$$
\begin{aligned}
\left(\mathscr{S}_{\phi}^{n} g\right)(u, v)= & \frac{1}{n^{2}} \sum_{0 \leq i+j \leq n-1} \Gamma\left(\frac{i+\frac{1}{2}}{n}, \frac{j+\frac{1}{2}}{n}, \frac{1}{n}\right) \\
& +\frac{1}{n^{2}} \sum_{0 \leq i+j \leq n-2} \Gamma\left(\frac{i+\frac{1}{2}}{n}, \frac{j+\frac{1}{2}}{n},-\frac{1}{n}\right) .
\end{aligned}
$$

Introducing additional zero terms, we have

$$
\left(\mathscr{S}_{\phi}^{n} g\right)(u, v)=\frac{1}{n^{2}} \sum_{i=0}^{n-1} \sum_{j=0}^{n-1}\left(\Gamma\left(\frac{i+\frac{1}{2}}{n}, \frac{j+\frac{1}{2}}{n}, \frac{1}{n}\right)+\Gamma\left(\frac{i+\frac{1}{2}}{n}, \frac{j+\frac{1}{2}}{n},-\frac{1}{n}\right)\right)
$$

As the function

$$
\Gamma(s, t, h)+\Gamma(s, t,-h)
$$

is even in $h$ and smooth for sufficiently small $h$, the asymptotic expansion (11) follows from Lemma 2 under the assumption (12). As $\Gamma^{(0,0, m)}(s, t, 0)$ is a polynomial of total degree $\leq m$ in $u$ and $v$ with coefficients depending on $s$ and $t$, it follows that $c_{k}(u, v)$ is a polynomial of total degree $\leq 2 k$ in $u$ and $v$. 
Now suppose the assumption (12) is not satisfied. There exists a partition of unity (cf. [5, Theorem 6.20, p. 147])

$$
\psi_{0}(u, v)+\psi_{1}(u, v)+\psi_{2}(u, v) \equiv 1,
$$

where $\psi_{0}, \psi_{1}, \psi_{2}$ are nonnegative smooth functions such that

$$
\begin{aligned}
& \psi_{0}(u, v)=0 \text { if } u+v \geq \frac{3}{4} \\
& \psi_{1}(u, v)=0 \text { if } u \leq \frac{1}{4} \\
& \psi_{2}(u, v)=0 \text { if } v \leq \frac{1}{4}
\end{aligned}
$$

We have

$$
\mathscr{S}_{\phi}^{n} g=\mathscr{S}_{\phi}^{n}\left(\psi_{0} g\right)+\mathscr{S}_{\phi}^{n}\left(\psi_{1} g\right)+\mathscr{S}_{\phi}^{n}\left(\psi_{2} g\right)
$$

Let

$$
\begin{aligned}
& g_{0}(u, v):=\left(\psi_{0} g\right)(u, v), \\
& g_{1}(u, v):=\left(\psi_{1} g\right)(1-u-v, v), \\
& g_{2}(u, v):=\left(\psi_{2} g\right)(u, 1-u-v) .
\end{aligned}
$$

Then

$$
\begin{aligned}
\left(\mathscr{S}_{\phi}^{n} g\right)(u, v)= & \left(\mathscr{S}_{\phi}^{n} g_{0}\right)(u, v)+\left(\mathscr{S}_{\phi}^{n} g_{1}\right)(1-u-v, v) \\
& +\left(\mathscr{S}_{\phi}^{n} g_{2}\right)(u, 1-u-v),
\end{aligned}
$$

and the function $g_{j}(u, v), j=1,2,3$, is smooth on $\sigma$ and

$$
g_{j}(u, v)=0 \text { if } u+v \geq \frac{3}{4} .
$$

Hence, according to the first part of the proof, $\mathscr{S}_{\phi}^{n} g_{j}$ admits an asymptotic expansion in even powers of $\frac{1}{n}$. The asymptotic expansion for $\mathscr{S}_{\phi}^{n} g$ can thus be obtained as the sum of the asymptotic expansions of the terms of the righthand side of (13).

Suppose $\phi$ is as in Theorem 1 and $f(x, y, z)$ is a smooth function on an open set containing $S=\phi(\sigma)$. Then $g(u, v)=(f \circ \phi)(u, v)$ is smooth on $\sigma$, and by Theorem 1

$$
\mathscr{S}_{\phi}^{n}(f \circ \phi)
$$

admits an asymptotic expansion in even powers of $\frac{1}{n}$. By (2) the dominant term of this expansion is the surface integral of $f$ over $S$. The modified trapezoidal rule (3) and the modified midpoint rule (4) thus have an asymptotic error expansion in even powers of $\frac{1}{n}$.

\section{BIBLIOGRAPHY}

1. Kurt Georg, Approximation of integrals for boundary element methods, SIAM J. Sci. Statist. Comput. 12 (1991), 443-453.

2. Kurt Georg and Johannes Tausch, Some error estimates for the numerical approximation of surface integrals, Math. Comp. 62 (1994), 755-763. 
3. J.N. Lyness, Quadrature over curved surfaces by extrapolation, Math. Comp. 63 (1994), 727740.

4. __ Quadrature over a simplex, SIAM J. Numer. Anal. 15 (1978), 122-133 and 870-877.

5. W. Rudin, Functional analysis, McGraw-Hill, New York, 1973.

Department of Computer Science, Katholieke Universiteit Leuven, CelestijnenlaAn 200 A, B-3001 LeUVEN, Belgium

E-mail address, P. Verlinden: pierreøcs.kuleuven.ac.be

E-mail address, R. Cools: ronaldecs.kuleuven.ac.be 